\title{
Phospholipid composition and kinetics in different endobronchial fractions from healthy volunteers
}

\author{
Ahilanandan Dushianthan ${ }^{1,2,3^{*}}$, Victoria Goss ${ }^{1,2}$, Rebecca Cusack ${ }^{1,2,3}$, Michael PW Grocott ${ }^{1,2,3}$ \\ and Anthony D Postle $e^{1,2}$
}

\begin{abstract}
Background: Alterations in surfactant phospholipid compositions are a recognized feature of many acute and chronic lung diseases. Investigation of underlying mechanisms requires assessment of surfactant phospholipid molecular composition and kinetics of synthesis and turnover. Such studies have recently become possible in humans due to the development of stable isotope labelling combined with advances in analytical methods in lipidomics. The objectives of this study are to compare phospholipid molecular species composition and phosphatidylcholine synthesis and turnover in surfactant isolated from various endobronchial compartments in healthy adults.
\end{abstract}

Methods: Healthy adults $(\mathrm{N}=10)$ were infused with methyl- $\mathrm{D}_{9}$-choline chloride and samples of induced sputum, tracheal wash and small volume bronchoalveolar lavage fluid were obtained subsequently at intervals up to 96 hours. Surfactant phospholipid composition and incorporation of stable isotope into surfactant phosphatidylcholine were determined by electrospray ionisation mass spectrometry.

Results: While molecular species compositions of phospholipids were similar for all three sample types, dipalmitoylphosphatidylcholine content was highest in lavage, intermediate in tracheal wash and lowest in sputum. Methyl- $\mathrm{D}_{9}$-choline incorporation into surfactant phosphatidylcholine was lower for sputum at 24 hours but reached equilibrium with other sample types by 48 hours. Fractional methyl- $\mathrm{D}_{9}$-dipalmitoylphosphatidylcholine incorporation for all sample types was about $0.5 \%$ of the endogenous composition. Lysophosphatidylcholine enrichment was twice than that of phosphatidylcholine.

Conclusions: Tracheal secretions may be of value as a surrogate to assess bronchoalveolar lavage fluid surfactant molecular composition and metabolism in healthy people. Despite minor differences, the phospholipid molecular composition of induced sputum also showed similarities to that of bronchoalveolar lavage fluid. Detailed analysis of newly synthesized individual phosphatidylcholine species provided novel insights into mechanisms of surfactant synthesis and acyl remodelling. Lysophosphatidylcholine methyl- $D_{9}$ incorporation patterns suggest that these species are secreted together with other surfactant phospholipids and are not generated in the air spaces by hydrolysis of secreted surfactant phosphatidylcholine. Application into patient populations may elucidate potential underlying pathophysiological mechanisms that lead to surfactant alterations in disease states.

Keywords: Surfactant, Phosphatidylcholine, Deuteriated choline, Stable isotopes, Isotope labelling, Mass spectrometry

\footnotetext{
* Correspondence: adushianthan@gmail.com

${ }^{1}$ NIHR Respiratory Biomedical Research Unit, University Hospital Southampton NHS Foundation Trust, Southampton SO16 6YD, UK

2Integrative Physiology and Critical IIIness Group, Clinical and Experimental Sciences, Sir Henry Wellcome Laboratories, Faculty of Medicine, University of Southampton, Southampton SO16 6YD, UK

Full list of author information is available at the end of the article
} 


\section{Background}

Continued synthesis and secretion of pulmonary surfactant is critically important for maintenance of optimal lung function throughout life. While primary surfactant deficiency in the lungs of preterm infants is widely acknowledged as a major cause of neonatal respiratory distress syndrome, secondary surfactant deficiency contributes to the pathology of many respiratory disorders of the mature lung including acute lung injury (ALI)/ acute respiratory distress syndrome (ARDS), asthma and cystic fibrosis [1]. Described mechanisms for surfactant dysfunction in respiratory diseases include inhibition by plasma proteins such as fibrinogen in oedema fluid, impaired synthesis and secretion by type II alveolar epithelial (ATII) cells and hydrolysis or oxidation of secreted surfactant [2]. Differentiating between these potential mechanisms is problematical in the clinical setting. Acquiring samples of alveolar and/or bronchial secretions for analysis of surfactant function or composition typically involves invasive bronchoscopic and bronchoalveolar lavage (BAL) procedures [3]. Additionally, analysis of concentrations and compositions of surfactant components such as surfactant proteins or phospholipids provides no information about processes of surfactant synthesis, secretion or turnover $[4,5]$. Availability of such information has considerable potential clinical implications, both for better understanding mechanisms of lung disease and for optimizing treatments for individual patients. Consequently, in this study we have assessed the molecular specificity of surfactant phospholipids extracted from small volume bronchoalveolar lavage fluid (BALF), tracheal wash (TW) and induced sputum (IS), representing secretions from various endobronchial compartments. In addition, we employed an in vivo stable isotope labelling strategy to monitor the incorporation of methyl$\mathrm{D}_{9}$-choline chloride into the phosphatidylcholine (PC) and lysophosphatidylcholine (LPC) fractions of the three different sample types. PC, especially the disaturated dipalmitoyl species (PC16:0/16:0), is the major surface active component of surfactant phospholipid. This stable isotope methodology enables the assessment of both the rate of synthesis and secretion of individual molecular species of surfactant PC and the specificity of fatty acyl remodelling mechanisms involved in their synthesis. This detailed analysis not only provides important information about mechanisms of surfactant PC synthesis and secretion, but comparison of fractional incorporation rates between sample types can also demonstrate the time required for newly secreted alveolar surfactant to transit to the upper airways.

\section{Methods}

Materials

Methyl-D - -choline chloride was from Cambridge Isotopes (CK Gases, Ibstock, UK); dimyristoylphosphatidylcholine
(PC14:0/14:0), heptadecyllysophosphatidylcholine (LP C17:0) and dimyristoylphosphatidylglycerol (PG14:0/14:0) were from Avanti Polar Lipids (Alabaster, USA). Solvents of HPLC quality from Fisher Scientific, UK.

\section{Study population}

Ten healthy volunteers without pre-existing lung diseases were recruited. All were non-smokers and had a normal medical examination including spirometry. Subjects with recent (within and up to 4 weeks) history of upper or lower respiratory tract infections were excluded. The age range was $18-36$ with a median age of 26 . The study protocol was approved by national ethics committee (South Central, Berkshire 11/SC/0185) and the University Hospital Southampton Research and Development Department. Informed consent was obtained from all healthy volunteers prior to the enrolment.

\section{Methyl- $D_{9}$ choline chloride}

Choline is an essential nutrient and a major constituent of the phospholipid fraction of pulmonary surfactant. Deuteriated choline (methyl- $\mathrm{D}_{9}$ choline chloride) is a stable isotope of choline, which can be used to trace phospholipid synthetic pathways. Recruited volunteers were intravenously infused with methyl- $\mathrm{D}_{9}$ choline chloride $(3.6 \mathrm{mg} / \mathrm{kg}$ body weight) for a period of 3 hours in accordance with a previous protocol [6].

\section{Induced sputum, tracheal wash and small volume BALF}

Sputum was induced by nebulised $4.5 \%$ hypertonic saline [7]. The induction was performed up to 20 minutes and stopped after sufficient material was obtained ( $2 \mathrm{mls})$. There were no events of bronchospasm or significant drop $(>15 \%)$ in peak flow during or after the process. Induced sputum was immediately mixed with $5 \mathrm{mls}$ of phosphate buffered saline (PBS) and transferred to ice. Tracheal wash and small volume broncholaveolar lavage samples were obtained by a fibre-optic bronchoscope performed under local anaesthesia without pre-medication. The bronchoscope was passed through mouth with the application of topical lidocaine (maximum of $4 \mathrm{mls} 2 \% \mathrm{w} / \mathrm{v}$ above vocal cords and $8 \mathrm{mls} 1 \% \mathrm{w} / \mathrm{v}$ below vocal cords. Ten mls of warmed saline $\left(37^{\circ} \mathrm{C}\right)$ was applied at the distal end of a right lower lobe distal segmental bronchus and was suctioned. A greater than $50 \%$ recovery was deemed to be adequate and if there was $<50 \%$ recovery, then a further $10 \mathrm{mls}$ of warmed saline was applied. TW samples were obtained by flushing the trachea with $10 \mathrm{ml}$ s warmed saline with subsequent suction. The subsequent BALF sampling was performed from a left lower lobe distal segmental bronchus next day. There was no significant decline in the $\mathrm{FEV}_{1}$ noted after the bronchoscopy. IS, BALF and TW samples were filtered through a $100 \mu \mathrm{m}$ mesh cell strainer (BD Falcon) and centrifuged at $400 \times \mathrm{g} \times 10$ 
minutes at $4^{\circ} \mathrm{C}$ to remove cells and debris. The supernatant was aspirated and stored at $-80^{\circ} \mathrm{C}$.

\section{Phospholipid extraction}

Internal standards of $1 \mathrm{nmol}$ dimyristoyl-PC (PC14:0/ 14:0), $0.1 \mathrm{nmol}$ heptadecyl_LPC (LPC17:0), $0.2 \mathrm{nmol}$ of dimyristoyl-PG (PG14:0/14:0) were added to all samples. Phospholipid fraction was extracted by modified Bligh and Dyer method [8]. Briefly, $800 \mu \mathrm{l}$ of sample and the addition of chloroform: methanol: water ( $\mathrm{v} / \mathrm{v}$ 2:2:1) resulted in biphasic layer. The lower phospholipid rich layer was aspirated carefully and dried under nitrogen gas at $37^{\circ} \mathrm{C}$.

\section{Mass spectrometry analysis}

Phospholipids were analyzed using a Xevo triple quadrupole mass spectrometer with electrospray ionisation interface (Walters, UK). Dried lipid extracts were dissolved in methanol:butanol:water:25\% $\mathrm{NH}_{4}(6: 2: 1.6: 0.4 \mathrm{v} / \mathrm{v})$ and delivered by direct infusion at $8 \mu \mathrm{l} / \mathrm{min}$. PC, LPC and sphingomyelin $(\mathrm{SPH})$ species were identified by precursor scans of the phosphocholine head group fragment, quantifying endogenous PC and LPC species from the fragment of mass to charge ratio $(\mathrm{m} / \mathrm{z})+184$ and deuteriated PC and LPC species from the $\mathrm{m} / \mathrm{z}+193$ fragment. Phosphatidylglycerol (PG) and phosphatidylinositol (PI) species were quantified from the negative ionisation spectrum. The ion peaks were quantified using MassLynx software with an in-house Excel macro programmed in Visual Basic.

\section{Determination of SP-D}

The SP-D content was determined by enzyme- linked immunosorbent assay (ELISA). The plates (96-well Nunc MaxiSorp, Fisher Scientific) were coated with capture antibody rfhSP-D 1ug/100ul per well in carbonate binding buffer (Sigma-Aldrich) and incubated at $4^{\circ}$ overnight. Plates were washed three times (PBS/T 0.05\% (v/v) Tween 20) and blocked for one hour (PBS/T with $2 \% \mathrm{BSA}$ ) at room temperature. After further wash, samples and standards were incubated for one hour at room temperature. After washing again the plates were incubated with streptavidin horseradish peroxidise 1:10,000 (Sigma-Aldrich) for one hour at room temperature. The plates were developed with TMB (Sigma-Aldrich), reaction was stopped with $0.5 \mathrm{M}$ $\mathrm{H}_{2} \mathrm{SO}_{4}$ and plates were read at $450 \mathrm{~nm}$.

\section{Statistics}

The data are expressed as mean \pm standard deviation (SD). A two tailed paired Student's T-test or two way analysis of variance was performed with Bonferoni correction for multiple comparisons (Graph Pad Prism version 5.04) to compare groups. Correlation was assessed using Pearson coefficient.

\section{Results}

Ten healthy volunteers were recruited. All participants had sputum induction. One subject, however, was unable to tolerate bronchoscopy leaving BALF/TW analysis for the remaining nine participants. The clinical data and summary of the participant characteristics were listed in Table 1.

\section{Total phospholipid, phosphatidylcholine and SP-D concentrations}

The mean total phospholipid concentration in BALF was 64.4 (range 29.1-145.1) $\mathrm{nmol} / \mathrm{ml}$, TW 51.8 (range 13.5- 158.7) $\mathrm{nmol} / \mathrm{ml}$ and IS 8.0 (Range 3.2-14.1) nmol/ml. The total PC concentration in BALF was relatively high (49.9 range $18.6-121.8 \mathrm{nmol} / \mathrm{ml}$ ) followed by TW $(37.2$ range $8.5-134.7 \mathrm{nmol} / \mathrm{ml}$ ) compared to IS (5.8 range 2.4-10.5 nmol/ml). The mean SP-D concentration was 30.3 (range 9.9-67.6) ug/ml for BALF, 24.4 (range 11.8-48.1) $\mathrm{ug} / \mathrm{ml}$ for TW and 17.5 (range 1.5-85.0) ug/ml for IS. The ratio of total SP-D/PC was much higher for IS, suggesting the possibility of additional secretion of SP-D from airway Clara cells (Table 2).

\section{Endogenous lipid compositions Phospholipid classes}

The fractional phospholipid composition was investigated by measuring the relative proportions of total PC, PG, PI, SPH and LPC, each determined as the sum of these individual molecular species. Phosphatidylethanolamine (PE) and phosphatidylserine (PS) were present at low concentrations and would have required additional analytical scans to assess molecular composition and consequently, these components are not presented here. PC (75\%) followed by PG (13\%) were the most abundant phospholipids. Although IS had a fractional increase in LPC and SPH, the phospholipid molecular composition was comparable among all sample types without any statistical difference (Figure 1).

\section{Molecular composition of phospholipid classes}

ESI/MS enabled a comprehensive analysis of molecular compositions of PC, LPC and SPH using positive and PG and PI with negative conditions. In BALF, di-saturated

\section{Table 1 Subject characteristics}

\begin{tabular}{lc}
\hline \multicolumn{2}{c}{ Characteristics } \\
\hline Age (Range) & $26(18-36)$ \\
M:F & $6: 4$ \\
FEV $_{1}$ (L) & $4.22 \pm 0.91^{*}$ \\
Weight (kg) & $78 \pm 12^{*}$ \\
Choline dose infused (mgs) & $275 \pm 54^{*}$ \\
BALF Volume recovery (\%) & $42 \pm 20^{*}$ \\
TW Volume recovery (\%) & $31 \pm 12^{*}$ \\
\hline
\end{tabular}

$M$, Male; $F$, Female; $F E V_{1}$, Forced expiratory volume in 1 second; BALF, Bronchoalveolar lavage fluid; $T W$, Tracheal wash. *Data expressed as mean \pm SD. 
Table 2 Concentrations of total phospholipid, phosphatidylcholine and surfactant protein D

\begin{tabular}{llll}
\hline & BALF & TW & IS \\
\hline Total PL $(\mathbf{n m o l} / \mathbf{m l})$ & $64.4 \pm 37.1(29.1-145.1)$ & $51.8 \pm 44.6(13.5-158.7)$ & $+8.0 \pm 3.8(3.2-14.1)$ \\
Total PC $(\mathbf{n m o l} / \mathbf{m l})$ & $49.9 \pm 32.8(18.6-121.8)$ & $37.2 \pm 39.1(8.5-134.7)$ & $+5.8 \pm 2.9(2.4-10.5)$ \\
SP-D $(\boldsymbol{\mu g} / \mathbf{m l})$ & $30.3 \pm 16.6(9.9-67.6)$ & $24.4 \pm 13.9(11.8-48.1)$ & $* 17.5 \pm 25.3(1.5-85.0)$ \\
SP-D:PC & 0.61 & 0.66 & 3.0 \\
\hline
\end{tabular}

$B A L F$, Bronchoalveolar lavage fluid; $T W$, Tracheal wash; $I S$, Induced sputum; $P L$, Phospholipid; $P C$, Phosphatidylcholine; $S P-D$, Surfactant protein $D .{ }^{*} P<0.05,{ }^{\dagger} P<0.01$ (paired Student's t-test) when compared with BALF, data expressed as mean $\pm S D$.

PC16:0/16:0 was the dominant PC accounting for more than $50 \%$ of total PC. This was followed by PC16:0/18:1 (13\%), PC16:0/16:1 (9\%), PC16:0/14:0 (9\%) and PC16:0/ 18:2 (6\%). These findings were consistent with previously published data [9]. When BALF PC composition was compared with other recovery methods, significantly lower proportions of PC16:0/16:0 were noted in both TW and IS. Additionally, IS also had significantly lower proportions of $\mathrm{PC} 16: 0 / 14: 0$ and $\mathrm{PC} 16: 0 / 16: 1$ and higher composition of PC16:0/18:1 and PC18:0/18:2 compared to BALF (Table 3). These differences suggest the possibility of dilution of surfactant PC by phospholipids derived from non-alveolar origin in induced sputum.

PG molecular species mainly comprised PG16:0/18:1 (35\%), PG18:1/18:1 (20\%), PG18:0/18:1 (19\%) while PI was dominated by PI18:0/18:1 (22\%), PI16:0/18:1 (20\%) and PI18:1/18:1 (20\%). This finding of unsaturated molecular species enrichment among PG and PI species is consistent with previously published data $[9,10]$. There were no significant differences in PG and PI compositions between BALF and TW. However, small but significant

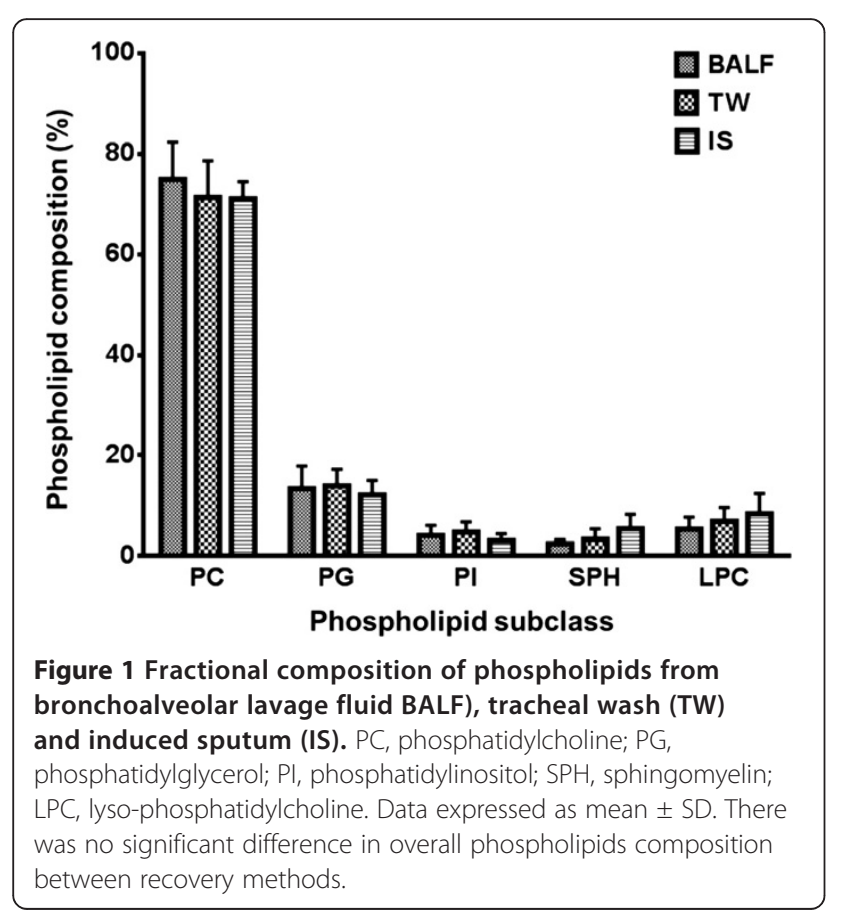

differences were noted in PG16:0/18:1 (3.3\% lower, $\mathrm{P}=$ 0.01), PG18:1/18:1 (3.1\% lower, $\mathrm{P}=0.02)$, and PI18:0/ $20: 4$ (4.8\% higher, $\mathrm{P}=0.02)$ in IS compared to BALF.

$\mathrm{SPH}$ species contain the same phosphocholine head group as PC and consequently can be readily detected by precursor scans of $\mathrm{m} / \mathrm{z}+184$ in ESI/MS. Major SPH species of BALF surfactant composed of SPH16:0 (65\%), SPH24:1 (20\%) and SPH24:0 (10\%). Other minor species (SPH16:1, SPH18:0, SPH18:1, SPH18:2 and SPH20:4) were detected at much lower abundance $(<3 \%)$. There were no significant differences in SPH molecular composition between BALF and TW. However, significant compositional differences with relative decrease in SPH16:0

Table 3 Phosphatidylcholine and lysophosphatidylcholine molecular species composition from three isolation methods

\begin{tabular}{lccc}
\hline \multicolumn{4}{c}{ Surfactant PC composition (\%) } \\
\hline PC Species & BALF & TW & IS \\
\hline PC16:0/14:0 & $8.8 \pm 0.9$ & $7.9 \pm 1.3$ & $\dagger 7.0 \pm 1.6$ \\
PC16:0a/16:0 & $2.6 \pm 0.4$ & $2.5 \pm 0.4$ & $3.0 \pm 0.6$ \\
PC16:0/16:1 & $9.0 \pm 1.5$ & $8.1 \pm 1.9$ & $\dagger 7.0 \pm 1.3$ \\
PC16:0/16:0 & $53.3 \pm 3.6$ & ${ }^{\ddagger} 48.7 \pm 6.4$ & ${ }^{\ddagger} 45.8 \pm 7.6$ \\
PC16:0/18:2 & $5.7 \pm 1.1$ & $6.9 \pm 1.8$ & ${ }^{\dagger} 7.6 \pm 1.8$ \\
PC16:0/18:1 & $12.6 \pm 1.6$ & $13.9 \pm 1.9$ & $\neq 15.3 \pm 2.4$ \\
PC16:0/20:4 & $1.4 \pm 0.4$ & $1.8 \pm 0.8$ & $1.9 \pm 0.7$ \\
PC18:1/18:2 & $1.6 \pm 0.5$ & $2.3 \pm 1.0$ & $2.9 \pm 0.9$ \\
PC18:0/18:2 & $2.7 \pm 1.0$ & $4.0 \pm 2.0$ & ${ }^{\ddagger} 5.4 \pm 2.0$ \\
PC18:0/18:1 & $1.3 \pm 0.6$ & $2.1 \pm 1.2$ & $2.9 \pm 1.3$ \\
PC18:1/20:4 & $0.4 \pm 0.2$ & $0.6 \pm 0.4$ & $0.5 \pm 0.2$ \\
PC18:0/20:4 & $0.6 \pm 0.4$ & $1.2 \pm 0.9$ & $0.9 \pm 0.4$ \\
\hline & Surfactant LPC composition \% & \\
\hline LPC Species & BALF & TW & IS \\
\hline LPC16:0 & $71.7 \pm 6.0$ & $\neq 65.7 \pm 9.2$ & $\neq 54.4 \pm 14.8$ \\
LPC18:2 & $6.4 \pm 1.4$ & $8.0 \pm 2.3$ & $\dagger 12.5 \pm 5.4$ \\
LPC18:1 & $13.3 \pm 2.5$ & $15.1 \pm 2.8$ & $\ddagger 21.6 \pm 6.2$ \\
LPC18:0 & $6.0 \pm 2.0$ & $8.0 \pm 3.3$ & $8.3 \pm 3.7$ \\
LPC20:4 & $2.6 \pm 1.8$ & $3.2 \pm 2.1$ & $3.2 \pm 2.2$ \\
\hline BALF, B & &
\end{tabular}

$B A L F$, Bronchoalveolar lavage fluid; $T W$, Tracheal wash; $I S$, Induced sputum; $P C$, Phosphatidylcholine; $L P C$, Lysophosphatidylcholine. ${ }^{\dagger} P<0.01,{ }^{\ddagger} P<0.001$. Data are expressed as \% (mean \pm SD) composition of total selected species at time points sampled. 
(2.9\% lower, $\mathrm{P}=0.0003)$ and increase in SPH24:1 $(1.8 \%$ higher, $\mathrm{P}=0.04$ ) was noted in IS compared to BALF.

Total PC and fractional PC methyl- $\mathrm{D}_{9}$ Incorporation Methyl- $\mathrm{D}_{9}$-choline incorporation was measured at two time points (24 and 48 hours) for BALF and TW and five time points $(8,24,48,72$ and 96 hours) for IS. In BALF, the total methyl- $\mathrm{D}_{9}$-choline incorporation into surfactant PC was $0.35 \pm 0.17 \%$ at 24 hours and $0.52 \pm$ $0.15 \%$ at 48 hours with a rate of incorporation of 0.011 $\pm 0.002 \%$ per hour until 48 hours (Figure 2). There was no difference in total PC methyl- $\mathrm{D}_{9}$-choline incorporation between BALF and TW. In addition, there was a positive correlation between BALF and TW for both time points $\left(\mathrm{r}^{2}=0.8344, \mathrm{P}<0.0001\right)$ for each individuals (Figure 2).

The methyl- $\mathrm{D}_{9}$-choline incorporation into total $\mathrm{PC}$ for IS was $0.13 \pm 0.03 \%$ at 8 hours, showed a linear incorporation until 48 hours $\left(\mathrm{r}^{2}=0.9984, \mathrm{P}=0.02\right)$ at a rate of $0.012 \pm 0.0005 \%$ per hour and remained relatively stable between 48-96 hours. The fractional incorporation into IS PC16:0/16:0 $(0.17 \pm 0.08 \%)$ was significantly lower $(\mathrm{P}<0.05)$ at $24 \mathrm{~h}$ than that into either BALF $(0.31 \pm$ $0.17 \%)$ or TW $(0.29 \pm 0.15 \%)$ (Figure 2$)$. However, fractional incorporation into IS PC16:0/16:0 reached equilibrium with other sample types at 48 hours. Although there was a positive correlation between BALF and IS for both time points $\left(\mathrm{r}^{2}=0.3572, \mathrm{P}=0.03\right)$, this association was much weaker than that for TW and BALF (Figure 2).

\section{Molecular specificity of $D_{9}$-labelled PC species}

The fractional composition of newly synthesized PC varied considerably between sample type and with time. The proportion of newly synthesized PC present as PC16:0/16:0 was consistently lower than that of endogenous PC composition for all sample types at 24 hours (Figure 3 ). In contrast, at 48 hours only IS still exhibited a lower proportion of newly synthesized compared with endogenous PC16:0/ 16:0. Inspecting the molecular specificity of PC synthesis in more detail for TW (Table 4), PC16:0/16:0 was the single molecular species significantly different from BALF at both 24 hours and 48 hours. The pattern of newly synthesized PC in IS was very different from BALF, with a total of six significantly different components at 24 hours and still two at 48 hours (Table 4).

\section{LPC - Composition and methyl- $D_{9}$-choline incorporation}

The LPC composition mainly consisted of LPC16:0 (72\%), LPC 18:1 (13\%) and LPC18:2 (6\%) (Table 3). Overall total LPC methyl- $\mathrm{D}_{9}$-choline incorporation was nearly twice than total PC incorporation for both time points (24 and 48 hours). Furthermore, LPC16:0 methyl$\mathrm{D}_{9}$-choline incorporation was twice as that of methyl- $\mathrm{D}_{9}$-labelled PC16:0/16:0 and correlated positively $\left(r^{2}=0.9318\right.$,
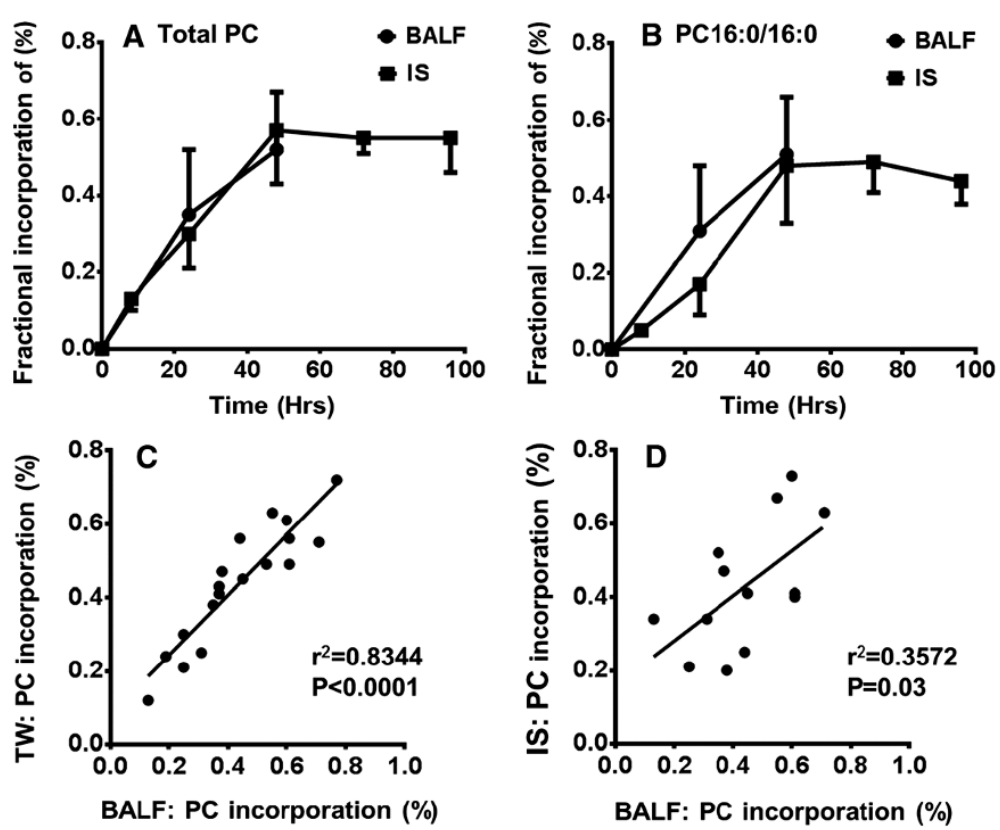

Figure 2 Fractional incorporation of methyl- $\mathrm{D}_{9}$-choline into total phosphatidylcholine (A) and PC16:0/16:0 (B) from bronchoalveolar lavage fluid and induced sputum. Comparison of methyl- $\mathrm{D}_{9}$ incorporation into total $\mathrm{PC}$ between bronchoalveolar lavage and tracheal wash (C) and bronchoalveolar lavage and induced sputum (D). The incorporation data for tracheal wash is not shown here as it followed the exact same pattern as BALF. Results are calculated as from the abundance of methyl- $\mathrm{D}_{9}-\mathrm{PC}$ species expressed as a percentage of endogenous $\mathrm{PC}+$ methyl- $\mathrm{D}_{9}-\mathrm{PC}$ species (mean \pm SD. Incorporation into PC16:0/16:0 but not into total PC was significantly lower in IS than BALF at $24 \mathrm{~h}\left({ }^{*} \mathrm{P}<0.05\right)$ but not at $48 \mathrm{~h}$. (PC, phosphatidylcholine; BALF, bronchoalveolar lavage fluid; IS, induced sputum, $\mathrm{r}^{2}$-Pearson correlation coefficient). 


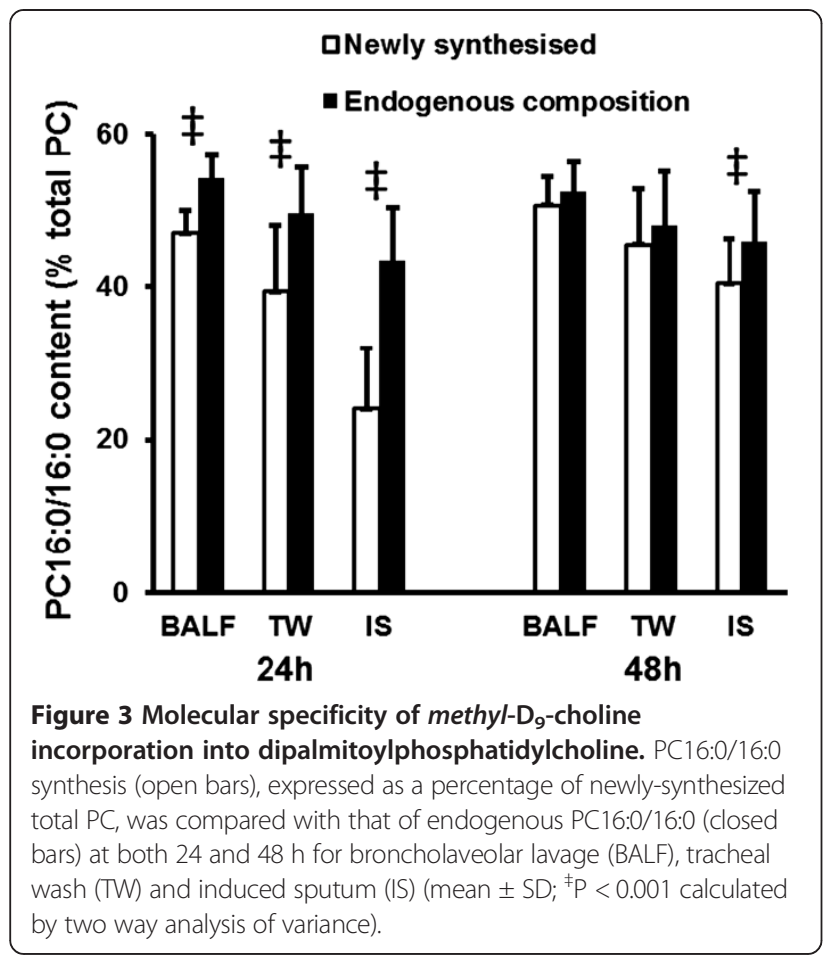

$\mathrm{P}<0.0001$ ) at both time points (Figure 4). However, the LPC 16:0 methyl- $\mathrm{D}_{9}$-labelling was much lower $(\sim 50 \%)$ compared to other LPC species.

\section{Discussion}

This study demonstrates for the first time the molecular compositions of various bronchoalveolar compartments in human model and the surfactant molecular PC kinetics from all these compartments. The results showed that surfactant extracted by TW closely resembled that of BALF. As ATII cells are the only source of pulmonary surfactant phospholipids, the significant positive correlation of methyl- $\mathrm{D}_{9}$-choline incorporation between BALF and TW suggests the mucocillary transit time of surfactant up along the airways had little significant impact on surfactant PC kinetics. Our analysis suggests that, despite the presence of already-secreted surfactant lining the small and large airway epithelium, a surfactant pool accessible from the upper airways by tracheal wash is in isotopic equilibrium with the alveolar surfactant pool.

Induced sputum had variable phospholipid composition and kinetics compared to BALF, possibly due to several reasons. Firstly, IS phospholipids may represent pools of surfactant with different temporal origins, with some material newly secreted from the alveolus combined with previously secreted surfactant embedded within the mucus layer. Secondly, sputum induction is a complex process and the exact origin of the induced sputum is not always known. Furthermore, the contamination from saliva and other non- surfactant phospholipids may have interfered with surfactant assessment. Although IS had qualitatively similar composition to that of alveolar surfactant [10], it may not be an ideal model to study alveolar surfactant metabolism. Thirdly, the relatively high content of sphingomyelin in induced sputum suggests proportion of IS phospholipid may be of cellular not surfactant origin. Such a cellular origin of IS phospholipid may become more significant in disease states characterized by increased airway inflammatory cell infiltration.

Surfactant PC is synthesized de-novo by the CDPcholine pathway. However, about $50 \%$ of $\mathrm{PC} 16: 0 / 16: 0$ is produced by acyl-remodelling mechanisms catalysed by

Table 4 Fractional composition of methyl- $D_{9}$-choline incorporation into newly synthesized PC at 24 and 48 hours

\begin{tabular}{|c|c|c|c|c|c|c|}
\hline \multicolumn{7}{|c|}{ Newly synthesized $D_{9}$-choline labelled molecular PC composition at 24 and 48 hours } \\
\hline \multirow[b]{2}{*}{ PC species (\%) } & \multicolumn{3}{|c|}{24 hours } & \multicolumn{3}{|c|}{48 hours } \\
\hline & BALF & TW & IS & BALF & TW & IS \\
\hline PC16:0/14:0 & $10.0 \pm 2.5$ & $7.8 \pm 2.8$ & $7.9 \pm 3.2$ & $8.8 \pm 1.0$ & $7.5 \pm 1.0$ & $7.3 \pm 1.9$ \\
\hline PC16:0a/16:0 & $2.6 \pm 0.6$ & $2.4 \pm 0.6$ & $4.0 \pm 2.9$ & $2.3 \pm 0.5$ & $2.3 \pm 0.5$ & $3.5 \pm 0.9$ \\
\hline PC16:0/16:1 & $10.0 \pm 2.5$ & $8.9 \pm 2.4$ & $7.03 \pm 2.0$ & $9.3 \pm 1.7$ & $8.1 \pm 2.1$ & $7.6 \pm 2.0$ \\
\hline PC16:0/16:0 & $46.9 \pm 3.0$ & ${ }^{\ddagger} 39.4 \pm 8.5$ & ${ }^{\ddagger} 22.5 \pm 7.9$ & $50.6 \pm 3.8$ & ${ }^{\ddagger} 45.4 \pm 7.3$ & ${ }^{\ddagger} 40.4 \pm 5.8$ \\
\hline PC16:0/18:2 & $6.9 \pm 1.8$ & $8.32 \pm 2.7$ & ${ }^{\dagger} 12.6 \pm 2.3$ & $6.1 \pm 1.4$ & $8.0 \pm 2.4$ & $8.3 \pm 0.9$ \\
\hline PC16:0/18:1 & $14.5 \pm 1.7$ & $17.5 \pm 2.8$ & $* 18.2 \pm 4.5$ & $13.9 \pm 1.9$ & $14.9 \pm 1.8$ & $15.9 \pm 2.2$ \\
\hline PC16:0/20:4 & $1.5 \pm 0.6$ & $2.2 \pm 0.9$ & $4.5 \pm 1.7$ & $1.5 \pm 0.5$ & $2.0 \pm 0.7$ & $2.1 \pm 1.0$ \\
\hline PC18:1/18:2 & $1.9 \pm 0.7$ & $3.1 \pm 1.5$ & $* 5.8 \pm 1.9$ & $2.0 \pm 0.6$ & $2.7 \pm 0.9$ & $3.5 \pm 1.4$ \\
\hline PC18:0/18:2 & $3.2 \pm 0.9$ & $5.4 \pm 2.8$ & ${ }^{\ddagger} 9.1 \pm 3.0$ & $2.8 \pm 1.1$ & $4.6 \pm 2.3$ & *6.0 61.9 \\
\hline PC18:0/18:1 & $1.3 \pm 0.6$ & $2.5 \pm 1.2$ & $* 4.7 \pm 2.3$ & $1.5 \pm 0.8$ & $2.3 \pm 1.8$ & $3.3 \pm 1.1$ \\
\hline PC18:1/20:4 & $0.5 \pm 0.2$ & $0.9 \pm 0.5$ & $1.5 \pm 1.1$ & $0.4 \pm 0.2$ & $0.7 \pm 0.4$ & $0.9 \pm 0.4$ \\
\hline PC18:0/20:4 & $0.7 \pm 0.4$ & $1.7 \pm 0.9$ & $2.1 \pm 0.9$ & $0.7 \pm 0.5$ & $1.3 \pm 1.1$ & $1.1 \pm 0.4$ \\
\hline
\end{tabular}

$\overline{B A L F}$, Bronchoalveolar lavage fluid; $T W$, Tracheal wash; IS, Induced sputum; $P C$, Phosphatidylcholine. ${ }^{*} \mathrm{P}<0.05,{ }^{\dagger} \mathrm{P}<0.01,{ }^{\ddagger} \mathrm{P}<0.001$ for $\mathrm{TW}$ and IS compared with BALF calculated by two way analysis of variance, data presented as mean \pm SD. 


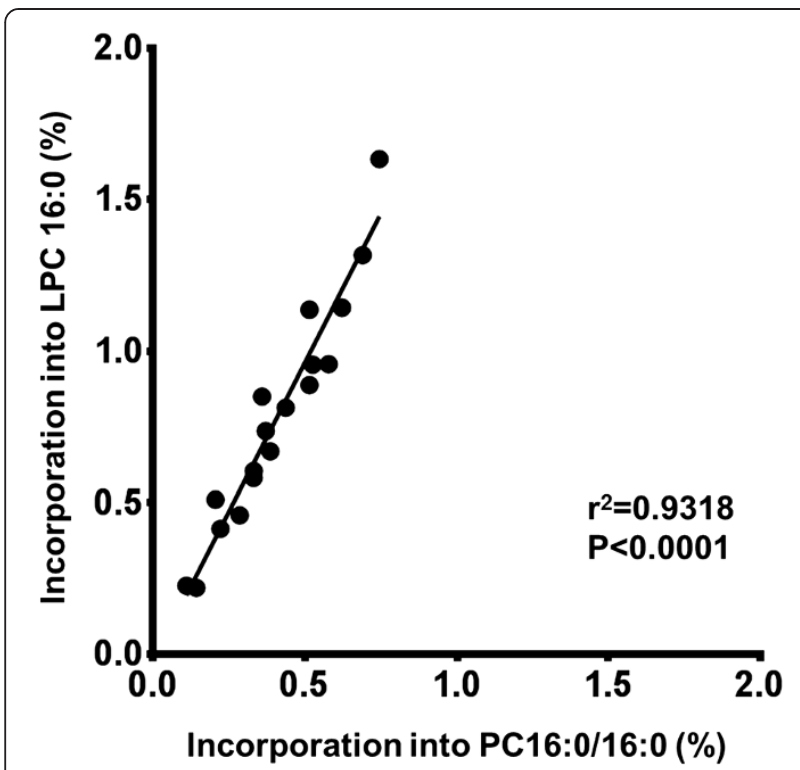

Figure 4 Comparison between methyl- $\mathrm{D}_{9}$ incorporation into PC16:0/16:0 and LPC16:0 isolated by bronchoalveolar lavage. $\left(r^{2}-\right.$ Pearson correlation coefficient).

the sequential actions of phospholipase- $\mathrm{A}_{2}$ and lysophosphatidylcholine acyltransferase activities [11]. It is widely assumed that surfactant PC synthesis and acyl-remodelling processes precede subsequent secretion [11]. However, our finding challenges this established concept. If the surfactant PC is secreted after acyl-remodelling and maturation process, the secreted surfactant PC16:0/16:0 compositions should reflect the endogenous composition at all time points. Our study shows that the proportion of methyl- $\mathrm{D}_{9}$-labelled $\mathrm{PC16:0/16:0}$ is equilibrated with endogenous composition only at 48 hours. This implies that a certain proportion of PC is secreted even before the acyl-remodelling mechanisms are complete.

This is the first to study to demonstrate the feasibility of assessing surfactant lyso-PC metabolism in-vivo in human subjects. This study shows that LPC16:0 is the principle lyso-PC in pulmonary surfactant, but that total LPC methyl- $\mathrm{D}_{9}$ incorporation was twice as that of total PC. The methyl- $\mathrm{D}_{9}$-incorporation of LPC16:0 is much higher than that of PC16:0/16:0 at both 24 and 48 hours. This finding challenges the paradigm of that the LPC is a formed by hydrolysis of secreted surfactant PC. The much higher fractional incorporation of LPC compared to PC precludes LPC coming from hydrolysis of newly synthesized PC and emphasizes the complexity of the underlying mechanisms of surfactant synthesis and secretion. These observations strongly support the possibility, at least in healthy individuals, that LPC is secreted together with the other surfactant phospholipid rather than simply being a consequence of hydrolysed PC. While phospholipase-mediated hydrolysis of secreted surfactant phospholipid remains a possibility in inflammatory lung disease, a further implication of our results, supported by the strong correlation of stable isotope label incorporations into LPC16:0 and PC16:0/16:0 (Figure 4) is that secreted LPC may be ultimately a consequence of acyl remodelling mechanisms within ATII cells.

Investigators have also used other stable isotopes such as ${ }^{13} \mathrm{C}$-glucose, ${ }^{13} \mathrm{C}$-fatty acids or deuteriated water to assess fractional synthetic rates (FSR) of saturated PC (SatPC) to characterise surfactant kinetics in neonates [12-14] and adults [15]. SatPC is the major PC surfactant component and a clinical marker of lung injury and surfactant derangement [16]. Measuring SatPC from deuteriated water enables maintenance of a steady state condition more easily, and therefore results can be compared between groups of patients with different clinical conditions. These novel studies provided significant insights into surfactant metabolism in humans but have some limitations. First, these methodologies initially used osmium tetroxide oxidation of unsaturated phospholipid to generate a SatPC fraction. Consequently, minimal information can be obtained about the molecular specificity of surfactant PC metabolism [17]. Second, the laborious sample preparation required for these studies has precluded the early diagnostic application of these methodologies to individuals with lung diseases. In contrast, the use of ESI-MS to monitor stable isotope incorporations requires minimal sample preparation and has the potential to generate diagnostic surfactant kinetic results in a time scale consistent with making clinical treatment decisions for individual patients with acute respiratory compromise.

In our study, tracheal secretions were accessed by the use of a bronchoscope. It is entirely plausible that a small amount of the BALF from distal alveolus may have contaminated the tracheal washings as both of these procedures were conducted in a single setting. If the compositional similarities were due to this contamination, one might have seen significant dilution effect in the magnitude of 1:10 reduction in the fractional phospholipid concentrations from tracheal wash. However, this was not the case and this absence of dilution effect indicate any contamination of tracheal wash from alveolar material is minimal.

Another limitation of this study was the measurements of absolute amounts of phospholipid subclasses and PC species may have been influenced by the variability in the sample recovery. Nevertheless it was reassuring to see overall compositional similarities from all endobronchial sampling. Furthermore, kinetic data regarding methyl- $\mathrm{D}_{9}$ incorporation patterns are unlikely to be influenced by this variability in recovery as the incorporation data was corrected for the endogenous material.

Acute lung injury and ARDS are characterized by significant quantitative and qualitative alterations in surfactant 
phospholipids composition [5]. Despite these findings, therapeutic attempts with exogenous surfactant remain unhelpful in this population [18]. The complexity of ARDS pathogenesis suggests several possible mechanisms for surfactant dysfunction, such as impaired synthesis and secretion, increased hydrolysis, proteolysis and oxidation or functional inhibition by infiltrating plasma proteins. However, in-vivo human models investigating such complex underlying mechanisms are lacking. Deficient understanding of the potential multiple mechanisms of aberrant surfactant metabolism in this disease cohort may in part explain the lack of anticipated clinical benefits from exogenous surfactant replacement strategies. Application of stable isotope studies in this population may possibly identify underlying phenotypes to characterise patients according to pathological mechanisms of surfactant dysfunction. Tracheal washings substituted for quantitative bronchoalveolar lavage may be an alterative for surfactant isolation in patients otherwise unable to tolerate invasive procedures without clinical compromise.

\section{Conclusions}

This study comprehensibly demonstrates the feasibility of performing stable isotope labelling to study surfactant phospholipid kinetics from bronchoalveolar compartments in healthy adults. Tracheal secretions were more closely resembled alveolar surfactant composition compared to induced sputum. Although several differences were noted in induced sputum phospholipid composition and turnover compared to BALF, the overall relative changes were small. This study illustrates the utility of various recovery methods to study in-vivo surfactant metabolism in humans which can be applied in disease states such as ARDS to possibly identify variation in surfactant metabolism among patients.

\section{Abbreviations \\ ALI: Acute lung injury; ARDS: Acute respiratory distress syndrome; ATII cells: Alveolar type II cells; BALF: Bronchoalveolar lavage fluid; ESI-MS: Electrospray ionisation mass spectrometry; IS: Induced sputum; LPC: Lysophosphatidylcholine; PC: Phosphatidylcholine; PG: Phosphatidylglycerol; PE: Phosphatidylethanolamine; PI: Phosphatidylinositol; PS: Phosphatidylserine; SatPC: Saturated phosphatidylcholine; SPH: Sphingomyelin; TW: Tracheal wash.}

\section{Competing interests}

ADP has no direct financial interest in the work presented in this study; his surfactant research programme is supported by kind donation of a therapeutic surfactant from Chiesi for a clinical trial. The remaining authors declare that they have no competing interests.

\section{Authors' contributions}

AD co-ordinated the study, contributed to the study design and the clinical and laboratory procedures, calculated the results and drafted the manuscript; VG co-ordinated the laboratory procedures and contributed to data interpretation and manuscript revision; RC contributed to the study design, ethics application, clinical procedures and manuscript revision; MPWG contributed to the study design, clinical procedures and manuscript revision; ADP contributed to the study design, data interpretation and manuscript revision. All authors read and approved the final manuscript.

\section{Acknowledgments}

This study is conducted by NIHR Respiratory Biomedical Research Unit and Anaesthesia and Critical Care Research Unit, University Hospital Southampton NHS Foundation Trust. We are grateful to Dr Rosie Mackay for guidance on the SP-D immunoassay and to the Wellcome Trust Clinical Research Facility, Southampton General Hospital for access to bronchoscopy and induced sputum collection and processing facilities.

\section{Sources of support}

AD was supported for consumables by a grant from the National Institute for Academic Anaesthesia; VG salary was supported from a NIHR Respiratory Biomedical Research Unit Grant, which also purchased the mass spectrometer.

\section{Author details}

${ }^{1} \mathrm{NIHR}$ Respiratory Biomedical Research Unit, University Hospital Southampton NHS Foundation Trust, Southampton SO16 6YD, UK. ${ }^{2}$ Integrative Physiology and Critical IIIness Group, Clinical and Experimental Sciences, Sir Henry Wellcome Laboratories, Faculty of Medicine, University of Southampton, Southampton SO16 6YD, UK. ${ }^{3}$ Anaesthesia and Critical Care Research Unit, CE 93, MP24, E-Level, Centre Block, University Hospital Southampton NHS Foundation Trust, Southampton SO16 6YD, UK.

Received: 27 June 2013 Accepted: 30 January 2014

Published: 1 February 2014

\section{References}

1. Griese M: Pulmonary surfactant in health and human lung diseases: state of the art. Eur Respir J 1999, 13:1455-1476.

2. Dushianthan A, Cusack R, Goss V, Postle AD, Grocott MP: Clinical review: exogenous surfactant therapy for acute lung injury/acute respiratory distress syndrome - where do we go from here? Crit Care 2012, 16:238.

3. Haslam PL: Bronchoalveolar lavage studies of pulmonary surfactant. Arch Bronconeumol 1994, 30:207-211.

4. Hallman M, Spragg R, Harrell JH, Moser KM, Gluck L: Evidence of lung surfactant abnormality in respiratory failure: study of bronchoalveolar lavage phospholipids, surface activity, phospholipase activity, and plasma myoinositol. J Clin Invest 1982, 70:673-683.

5. Günther A, Siebert C, Schmidt R, Ziegler S, Grimminger F, Yabut M, Temmesfeld B, Walmrath D, Morr H, Seeger W: Surfactant alterations in severe pneumonia, acute respiratory distress syndrome, and cardiogenic lung edema. Am J Respir Crit Care Med 1996, 153:176-184.

6. Bernhard W, Pynn CJ, Jaworski A, Rau GA, Hohlfeld JM, Freihorst J, Poets CF, Stoll D, Postle AD: Mass spectrometric analysis of surfactant metabolism in human volunteers using deuteriated choline. Am J Respir Crit Care Med 2004, 170:54-58.

7. Paggiaro PL, Chanez P, Holz O, Ind PW, Djukanović R, Maestrelli P, Sterk PJ: Sputum induction. Eur Respir J Suppl 2002, 37:3s-8s.

8. Bligh EG, Dyer WJ: A rapid method of total lipid extraction and purification. Can J Biochem Physiol 1959, 37:911-917.

9. Postle AD, Heeley EL, Wilton DC: A comparison of the molecular species compositions of mammalian lung surfactant phospholipids. Comp Biochem Physiol A Mol Integr Physiol 2001, 129:65-73.

10. Wright SM, Hockey PM, Enhorning G, Strong P, Reid KB, Holgate ST, Djukanovic R, Postle AD: Altered airway surfactant phospholipid composition and reduced lung function in asthma. J Appl Physiol 2000, 89:1283-1292

11. Batenburg JJ: Surfactant phospholipids: synthesis and storage. Am J Physiol 1992, 262:L367-L385.

12. Bunt JE, Zimmermann LJ, Wattimena JL, van Beek RH, Sauer PJ, Carnielli VP: Endogenous surfactant turnover in preterm infants measured with stable isotopes. Am J Respir Crit Care Med 1998, 157:810-814.

13. Cavicchioli P, Zimmermann L, Cogo PE, Badon T, Giordano G, Torresin M, Zacchello F, Carnielli VP: Endogenous surfactant turnover in preterm infants with respiratory distress syndrome studied with stable isotope lipids. Am J Respir Crit Care Med 2001, 163:55-60.

14. Cogo PE, Gucciardi A, Traldi U, Hilkert AW, Verlato G, Carnielli V: Measurement of pulmonary surfactant disaturated-phosphatidylcholine synthesis in human infants using deuterium incorporation from body water. J Mass Spectrom 2005, 40:876-881.

15. Simonato M, Baritussio A, Ori C, Vedovelli L, Rossi S, Dalla Massara L, Rizzi S, Carnielli VP, Cogo PE: Disaturated-phosphatidylcholine and surfactant 
protein-B turnover in human acute lung injury and in control patients. Respir Res 2011, 12:36.

16. Hillman NH, Kallapur SG, Pillow JJ, Nitsos I, Polglase GR, Ikegami M, Jobe AH: Inhibitors of inflammation and endogenous surfactant pool size as modulators of lung injury with initiation of ventilation in preterm sheep. Respir Res 2010, 11:151.

17. Goss V, Hunt AN, Postle AD: Regulation of lung surfactant phospholipid synthesis and metabolism. Biochim Biophys Acta 1831, 2013:448-458.

18. Meng H, Sun Y, Lu J, Fu S, Meng Z, Scott M, Li Q: Exogenous surfactant may improve oxygenation but not mortality in adult patients with acute lung injury/acute respiratory distress syndrome: a meta-analysis of 9 clinical trials. J Cardiothorac Vasc Anesth 2012, 26:849-856.

doi:10.1186/1471-2466-14-10

Cite this article as: Dushianthan et al:: Phospholipid composition and kinetics in different endobronchial fractions from healthy volunteers. BMC Pulmonary Medicine 2014 14:10

\section{Submit your next manuscript to BioMed Central and take full advantage of:}

- Convenient online submission

- Thorough peer review

- No space constraints or color figure charges

- Immediate publication on acceptance

- Inclusion in PubMed, CAS, Scopus and Google Scholar

- Research which is freely available for redistribution 